“(C) 2018 IEEE. Personal use of this material is permitted. Permission from IEEE must be obtained for all other uses, in any current or future media, including reprinting/republishing this material for advertising or promotional purposes, creating new collective works, for resale or redistribution to servers or lists, or reuse of any copyrighted component of this work in other works." 


\title{
Circularly-Polarized Antenna with Reconfigurable Broadside and Conical Beams Facilitated by a Mode Switchable Feed Network
}

\author{
Wei Lin, Hang Wong and Richard W. Ziolkowski
}

\begin{abstract}
A pattern-reconfigurable slot-augmented patch antenna is reported that radiates circularly-polarized (CP) fields and is switchable between a broadside and a conical radiating mode. Two novel design strategies are adopted to realize these advantageous performance characteristics. First, a mode switchable feed network which includes a set of eight L-probes was developed that facilitates the proper excitation of either the patch's $T M_{11}$ or $\mathbf{T} M_{21}$ mode and yields symmetric radiation patterns. Second, their operating bands are made to overlap by augmenting this patch with an annular slot. Consequently, the radiation pattern is easily switched between the broadside and the conical mode within the overlapping operational bandwidths. A prototype was fabricated and tested. The measured results are in good agreement with their simulated values, verifying the design concepts. The measured operating bandwidth, $7.8 \%$ from 2.45 to $2.65 \mathrm{GHz}$, was determined from the overlap of the impedance and AR bandwidths for both modes. The measured realized gain values are stable for both radiating states within this operational bandwidth, the broadside (conical) peak value being 8.5 dBic $(5.8 \mathrm{dBic})$. The $\mathrm{CP}$ pattern reconfigurability of this antenna with its favorable performance characteristics makes it an ideal candidate for many RFID and satellite communication applications.
\end{abstract}

Index Terms - Broadside and conical radiation patterns, circular polarization, L-probe feed network, patch antenna, pattern reconfigurable antenna

\section{INTRODUCTION}

Pattern-reconfigurable antennas have drawn much attention with the rapid development of modern wireless communication systems. For instance, previously reported antennas with pattern reconfigurability [1]-[9] can reshape their radiation patterns for many purposes. These include object detection, larger radiation coverage, and channel capacity enhancement in MIMO systems [10].

In this Communication, our efforts are focused on obtaining an advantageous pattern reconfigurability, i.e., switchable CP broadside and conical beams. This reconfigurability increases the $\mathrm{CP}$ radiation coverage, a highly desirable, beneficial property for RFID and satellite communications applications. For example, Fig. 1 presents a satellite communications application diagram that illustrates these benefits. A conical-beam CP antenna, which is mounted on the top of a vehicle moving on the earth, is quite suitable for communicating with geostationary satellites positioned at angles away from the geocentric zenith without any need for satellite tracking [11], [12]. Note that geostationary satellites are stationary and have a fixed geocentric zenith with respect to a specific area on the earth. Assuming that a satellite has a specified elevation angle away from that geocentric zenith, the maximum radiation of a conical-beam antenna mounted on an earth-based object can be designed to always

Manuscript received on Mar. 08, 2017; revised 02 Aug. 2017, ...

Wei Lin and Richard W. Ziolkowski are with the University of Technology Sydney, Global Big Data Technologies Centre, Ultimo NSW 2007, Australia (E-mail: wei.lin@uts.edu.au; Richard.Ziolkowski@uts.edu.au). R. W. Ziolkowski is also with the Department of Electrical and Computer Engineering, University of Arizona, Tucson, AZ 85721, USA.

Hang Wong is with the State Key Laboratory of Millimeter Waves, Department of Electronic Engineering, City University of Hong Kong, Hong Kong SAR, China (E-mail: hang.wong@cityu.edu.hk).

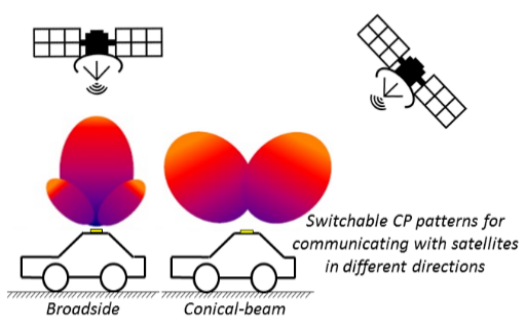

Fig. 1. An application diagram of a pattern-reconfigurable $\mathrm{CP}$ antenna with broadside and conical beams mounted on a vehicle moving on the earth that is communicating with a geostationary satellite.

point at the satellite no matter how the object rotates on the earth. Consequently, complicated satellite tracking can be avoided.

On the other hand, as Fig. 1 also depicts, there are cases in which the satellite is located directly above the vehicle. They occur, for example, in regions near the equator of the earth, such as in Singapore, Indonesia, and the large central region of Africa. A conical-beam pattern antenna is no longer useful because it operates with a null in its broadside direction. A CP antenna that radiates primarily in its broadside direction is needed. Consequently, it is clear that a CP antenna with switchable broadside and conical radiation patterns would be an excellent candidate for satellite communications all over the world.

Several efforts [13]-[16] have achieved reconfigurable systems that radiate both broadside (unidirectional) and conical (omnidirectional) beams. The main design technique is to excite different modes on the same antenna structure, each producing a distinctive radiation pattern. Unfortunately, all these antennas are linearly-polarized (LP). To date (and to the best of our knowledge) only one reported design [17] has realized a switchable broadside and conical beam CP antenna. It has two physically separate radiating systems to achieve both beam states. Its feeding network is inherently narrow-band and lossy. As a consequence, it has a narrow operating bandwidth $(1.6 \%)$ and produces small peak realized gain values (broadside mode: $4.0 \mathrm{dBic}$; conical mode: $2.0 \mathrm{dBic}$ ).

We present a pattern-reconfigurable $\mathrm{CP}$ antenna with switchable broadside and conical beams that is a simpler structure and has much better radiation performance than the one reported in [17]. Its design and operating principles are detailed in Section II. Its superior performance arises from two novel design strategies. First, an annular slot augmentation of its circular patch facilitates the presence of two modes and the overlap of their operational frequency bands. Second, a mode switchable feed network was developed that controls the excitation of this slot-augmented circular patch radiator. This network switches the amplitudes and phases of two sets of four Lprobe feed elements to properly excite the $\mathrm{TM}_{11}$ and $\mathrm{TM}_{21}$ modes of the radiator. The $\mathrm{TM}_{11}\left(\mathrm{TM}_{21}\right)$ mode produces the broadside (conical) CP radiation. The multiple probe system facilitates the generation of symmetric beams. Two key parametric design studies are summarized in Section III. As described in Section IV, a prototype was fabricated, assembled, and tested. The experimental results successfully demonstrate the design concept and are in good agreement with their simulated values. 

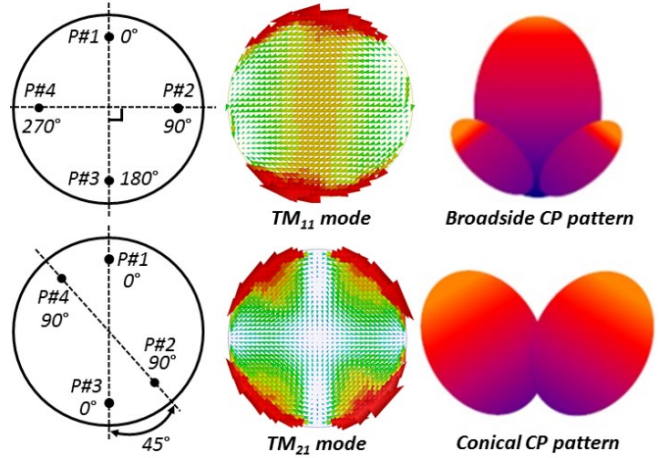

Fig. 2. Simulated $\mathrm{TM}_{11}$ and $\mathrm{TM}_{21}$ modes excited on a circular patch and the associated broadside and conical $\mathrm{CP}$ radiation patterns.

\section{Antenna Design And Operating Principles}

The concept of realizing switchable broadside and conical $\mathrm{CP}$ beams is based on the distinctive patterns radiated by the $\mathrm{TM}_{11}$ and $\mathrm{TM}_{21}$ modes excited on a circular patch antenna. As shown in Fig. 2, different locations and phases of the excitations are required to generate these two TM modes. These behaviors guided our mode switchable feed network design. The overlap of the operational bandwidths of these modes is based on augmenting the patch with an annular ring.

\section{A. Antenna configuration}

The antenna consists of three main parts as shown in Fig. 3. The key design parameters are listed in Table I. From its perspective view in Fig. 3(a) the antenna configuration consists of three circular dielectric substrate disks. They are labeled: Substrate patch, Substrate feeding, and Substrate_DC from the top to the bottom, respectively. The dielectric substrate is from Wangling Ltd and has a relative permittivity of 2.65 , loss tangent of 0.001 and $1.0 \mathrm{~mm}$ thickness. The four plastic screws shown are used to mechanically support for the three substrates. The top of the substrate_patch layer is the circular patch radiator. It has an annular slot etched in it.

The middle layer, Substrate feeding, shown in Fig. 3(b) contains the reconfigurable feed network. It consists of an output switchable network connected with L-shaped feed probes. There are one input (feed port) and four output ports. This network includes three Wilkinson power dividers. Microstrip lines are included and PIN diodes are integrated into them to achieve the reconfigurability, i.e, the switching between the different phases and locations of the excitations of the patch radiator. In addition, six L-shaped probes, $\mathrm{P} \# 1$ to $\mathrm{P} \# 6$, are connected to the output ports of the feed network as those excitations of the patch. Consequently, four L-probes are excited for each mode. Two additional suspended L-probes are also arranged in the configuration to ensure a symmetrical structure and, consequently, good CP radiation in both modes. There are RF chokes present to isolate the RF signal and prevent it from floating into the DC biasing lines. A 3D-printed fixture is attached on the top surface of the Substrate_feeding layer to secure the eight L-shaped probes.

Finally, the third substrate, Substrate_DC, is placed below the Substrate feeding layer with a vertical gap of $1.0 \mathrm{~mm}$. The dielectric material of this substrate is FR-4; it has a relative permittivity of 4.4 and a $1.0 \mathrm{~mm}$ thickness. The DC biasing lines that are needed to provide the DC voltages to the diodes are printed on its bottom side along with four biasing pads. These four pads are labeled as $D C \_$conical, DC_broadside, DC_ground\#1 and DC_ground\#2 as shown in Fig. 3(c). Note that a small region of the Substrate_DC layer is removed and filled with a $50 \Omega$ SMA connector to give the source access to the feed network. The biasing connections from the bottom to the top layer are illustrated and detailed in the subplot given in Fig. 3(c).

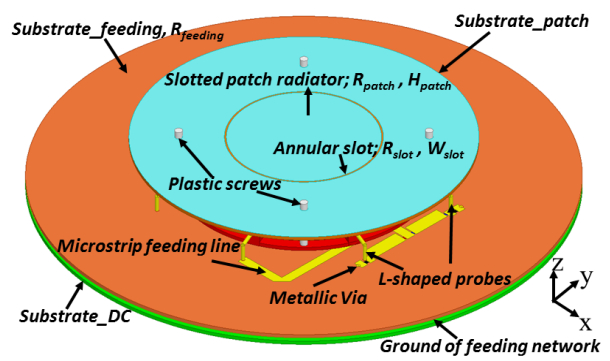

(a) 3D perspective view

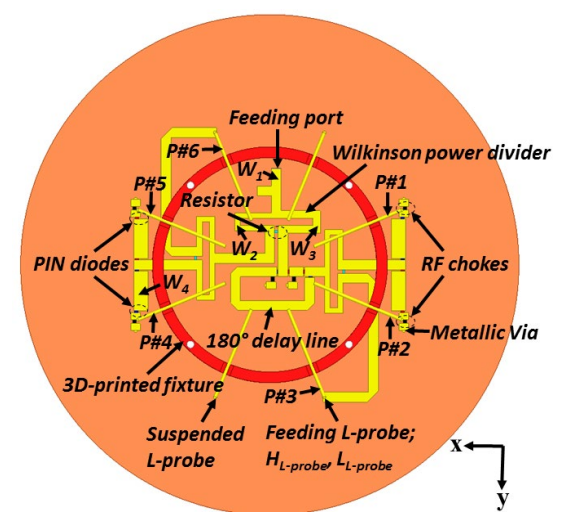

(b) Top view of the feed network without the patch being present

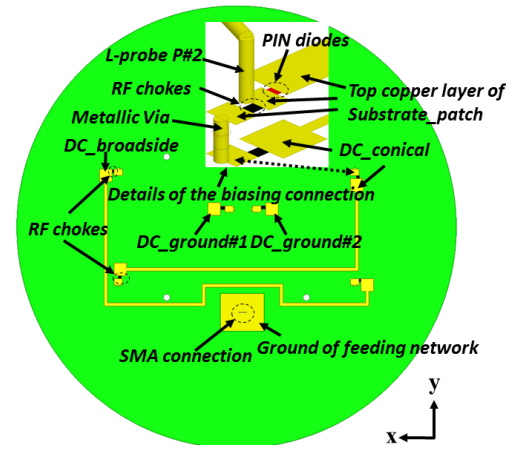

(c) Bottom view

Fig. 3. Antenna configuration. (a) Perspective view. (b) Top view of the feed network without patch. (c) Bottom view.

TABLE I

ANTENNA PARAMETERS (DIMENSIONS IN MILLIMETERS)

\begin{tabular}{clc}
\hline \hline Parameter & \multicolumn{1}{c}{ Description } & Value \\
\hline$R_{\text {patch }}$ & Radius of the slotted-circular patch & 44.0 \\
$R_{\text {slot }}$ & Radius of the annular slot on patch & 19.5 \\
$W_{s}$ & Width of the annular slot & 0.5 \\
$H_{\text {patch }}$ & Height of the patch above ground & 13.0 \\
$H_{L-\text { probe }}$ & Height of the L-probe & 5.0 \\
$L_{L-\text { probe }}$ & Length of the L-probe & 27.0 \\
$R_{\text {-probe }}$ & Radius of the L-probe from axis center & 40.0 \\
$R_{\text {feeding }}$ & Radius of the feed network & 70.0 \\
$G_{\text {diode }}$ & Gap of the PN diode & 0.4 \\
$G_{\text {inductor }}$ & Gap of the inductor as RF choke & 0.4 \\
$G_{\text {resistor }}$ & Gap of the isolation resistor & 3.5 \\
$W_{I}$ & Width of the 50 ohm microstrip line & 2.72 \\
$W_{2}$ & Width of part of the quarter-wave line & 1.5 \\
$W_{3}$ & Width of part of the quarter-wave line & 2.2 \\
$W_{4}$ & Width of the microstrip line for the feed & 4.0 \\
\hline \hline
\end{tabular}


B. Annular slot in the circular patch facilitates the overlap of the resonant frequencies of $T M_{11}$ and $T M_{21}$ modes

The slot-augmented patch design strategy is another key factor in the realization of the desired reconfigurable broadside and conical CP radiation behaviors. As discussed in [18], it is well-known that the resonance frequencies of the $\mathrm{TM}_{11}$ and $\mathrm{TM}_{21}$ modes of a traditional circular patch are different. The theoretical ratio of resonant frequencies of the fundamental mode $\mathrm{TM}_{11}$ and its higher order $\mathrm{TM}_{21}$ mode is $1.84 / 3.05=0.6$. However, if a thin annular slot is etched into the circular patch, the resonance frequency of the $\mathrm{TM}_{11}$ mode increases while that of the $\mathrm{TM}_{21}$ mode remains unchanged. Consequently, the bandwidth of the $\mathrm{TM}_{11}$ mode can be made to overlap with the $\mathrm{TM}_{21}$ mode; hence, the slot-augmented patch radiator will support the operation of both modes within the same frequency interval. To illustrate this point, the simulated current distributions on the patch radiator when their $\mathrm{TM}_{11}$ and $\mathrm{TM}_{21}$ modes are excited at $2.5 \mathrm{GHz}$ for several different antenna configurations are shown, respectively, in Figs. 4 and 5. These designs include a circular patch with and without the annular slot, and a smaller patch that is concentric with its surrounding slot.

The phases and locations of the excitations of the $\mathrm{TM}_{21}$ mode in Fig. 5 are the same as those shown in the bottom left subplot of Fig. 2. The simulated current distribution on the large patch without an annular slot indicates that the $\mathrm{TM}_{21}$ mode is well excited. The radius of this circular patch is $44.0 \mathrm{~mm}$, i.e., $0.37 \lambda_{0}$ at $2.5 \mathrm{GHz}, \lambda_{0}$ being the associated free-space wavelength. On the other hand, if a $0.5 \mathrm{~mm}$ wide, $19.5 \mathrm{~mm}$ radius annular slot is etched in the patch about its center, a similar current distribution for the $\mathrm{TM}_{21}$ mode results. The corresponding left-hand (LHCP) and right-hand (RHCP) circularly polarized gain patterns are also given in Fig. 4. They clearly indicate that both of these patch antennas produce equivalently strong LHCP radiation with a conical gain pattern and, in particular, that a thin annular slot on a circular patch will not affect the resonance frequency of the $\mathrm{TM}_{21}$ mode. In contrast, the $\mathrm{TM}_{21}$ mode is not supported well by the smaller $19.5 \mathrm{~mm}$ radius patch at the same frequency, resulting in a poor LHCP gain pattern.

In contrast, Fig. 4 shows the same simulation results when these antennas are excited at $2.5 \mathrm{GHz}$ with the $\mathrm{TM}_{11}$ mode arrangement shown in the upper left subplot of Fig. 2. The resonance frequency of the $\mathrm{TM}_{11}$ mode is much lower than $2.5 \mathrm{GHz}$. Consequently, one immediately observes that the $\mathrm{TM}_{11}$ mode is not excited well on the large patch without a slot being present. The corresponding broadside gain pattern is also poorly developed. However, when the annular slot is etched into the radiating patch, the $\mathrm{TM}_{11}$ mode current distribution appears on it and the broadside gain pattern is well developed at $2.5 \mathrm{GHz}$. The smaller patch case also shows a similar outcome. These results demonstrate that the slotted-patch antenna as an entity can support both the $\mathrm{TM}_{11}$ and $\mathrm{TM}_{21}$ modes in the same frequency interval. This feature is distinctly different from the system in [17] which employs two separate radiators with individual vertical probe excitations for each mode to achieve its reconfigurability.

\section{Realization of the reconfigurable feed network}

To switch between the proper excitations of both the $\mathrm{TM}_{11}$ and $\mathrm{TM}_{21}$ modes, PIN diodes were introduced between the transmission lines of the 1-to-4 Wilkinson power divider feed network. Note that the choice of four excitations for the slot-augmented circular patch radiator rather than just two is critical for this design. The radiation patterns of both modes will become asymmetrical and the cross polarization levels will increase noticeably if only two excitations are employed. Figure 6 shows the arrangement of these PIN diodes.
By controlling their ON/OFF status, different locations and phases of the four excitations are realized. Each feed combination corresponds to a particular mode excitation.

The PIN diodes Bar50-02L with 0403 surface mount packaging from Infineon Technologies were selected. They have excellent switching performance around $2.5 \mathrm{GHz}$. The measured characteristics and equivalent circuit model were reported previously [19]. To control the diode states, four DC bias voltages are needed. They are labeled as DC_broadside, DC_conical, DC_ground\#1 and DC_ground\#2. The total DC power consumption of each PIN diode is $12 \mathrm{~mW}$, i.e., $\mathrm{P}_{\mathrm{dc}}=\mathrm{I}^{2} \mathrm{R}=(100 \mathrm{~mA})^{2} \times 1.2 \Omega$ as calculated from its datasheet [20]. The detailed connections of these DC biases are presented in both Figs. 3(c) and 6.
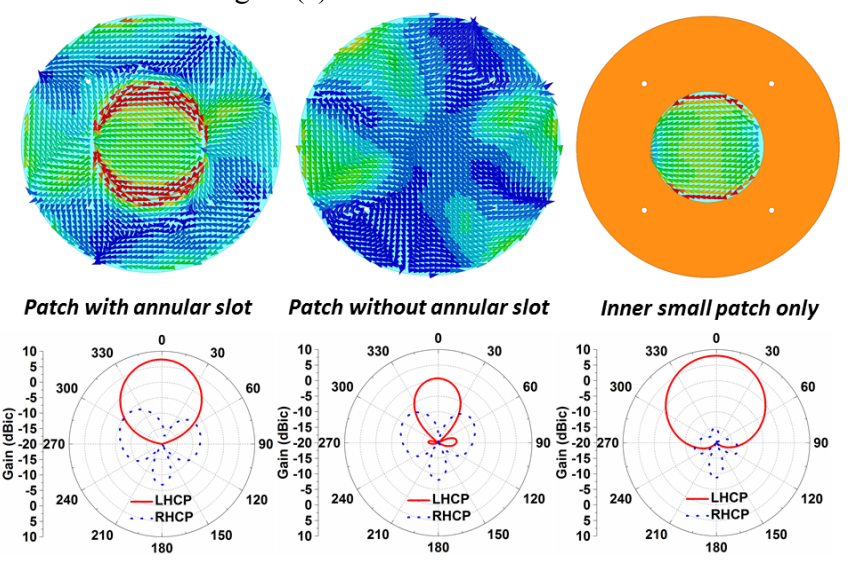

Fig. 4. Simulated current distributions on three different patch antenna structures and the corresponding LHCP and RHCP gain patterns when the $\mathrm{TM}_{11}$ mode is excited at $2.5 \mathrm{GHz}$.
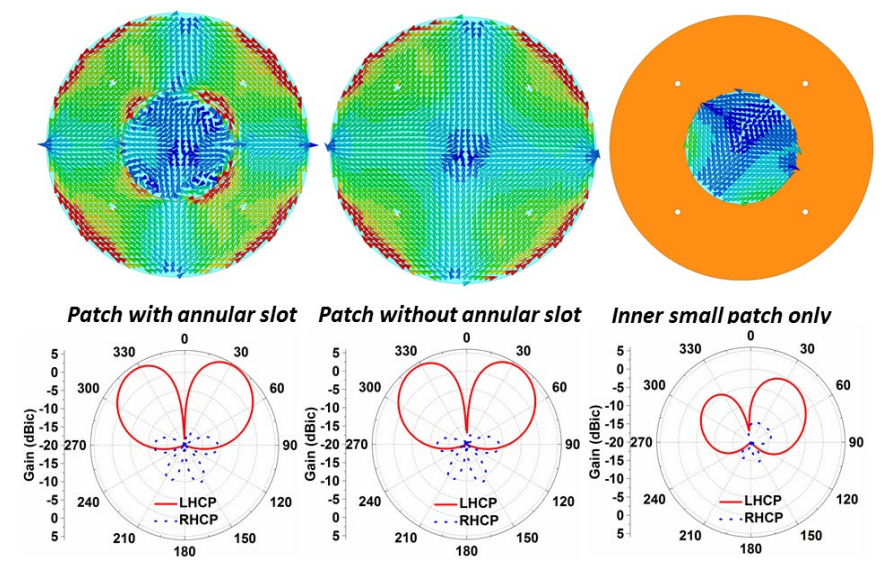

Fig. 5. Simulated current distributions on three different patch antenna structures and the corresponding LHCP and RHCP gain patterns when the $\mathrm{TM}_{21}$ mode is excited at $2.5 \mathrm{GHz}$.

From the bottom layer Substrate_DC, six metallic vias connect the bottom DC lines and the biasing pads on the upper layer of the feed network through inductors, which act as RF chokes. The Murata inductors Type: LQW18AN56NJ00 with 0603 packaging were selected. A complete characterization of these inductors is found in [21]. The DC bias configurations control the excitation of the patch modes. The two small patches connected with the L-probes P\#1 \& P\#4 and the diodes are biased with DC_broadside. Similarly, the two small patches connected with the L-probes $\mathrm{P} \# 2$ \& $\mathrm{P} \# 5$ and the diodes are biased with DC_conical. The half wavelength delay line is biased with DC_ground\#1. The last small patch is biased with DC_ground\#2. 


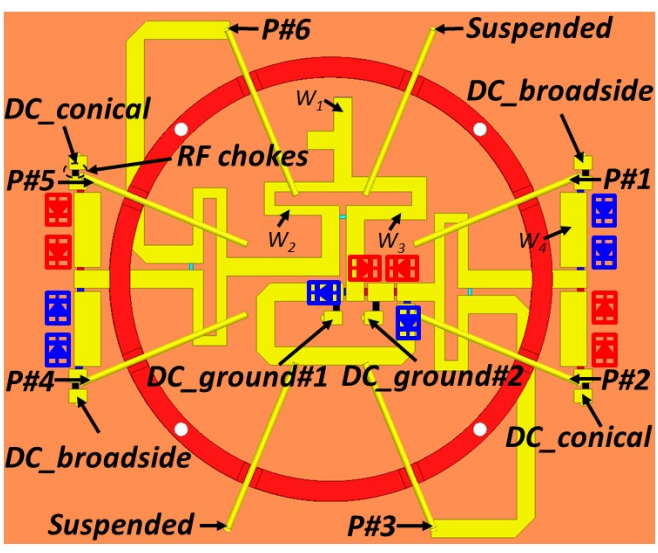

Fig. 6. Arrangement of the diodes and their DC biases.

If a $3 \mathrm{~V}$ DC voltage is applied to DC_broadside with this arrangement and DC_ground\#1 is set to ground with $0 \mathrm{~V}$, all the blue diodes will be turned on and all red diodes will be off. As a result, the $\mathrm{TM}_{11}$ mode will be excited. The L-probes P\#1, P\#3, P\#4 and P\#6 are excited with a clockwise sequential phase delay, generating the broadside CP radiation pattern as indicated in Table II. The current magnitudes of the two unfed L-probes and the two suspended L-probes are much lower than those on the feed probes. The current distributions on the feed network realized in this manner are shown in Fig. 7. On the other hand, if a $3 \mathrm{~V}$ DC voltage is applied to DC_conical and DC_ground\#2 is set to ground with $0 \mathrm{~V}$, all the red diodes will be turned on and the blue diodes will be off. As a result, the $\mathrm{TM}_{21}$ mode will be excited. The L-probes $\mathrm{P} \# 2, \mathrm{P} \# 3, \mathrm{P} \# 5$ and $\mathrm{P} \# 6$ are excited with the phases: $90^{\circ}, 0^{\circ}, 90^{\circ}$ and $0^{\circ}$, respectively, generating the conical CP radiation patterns as indicated in Table II. Again, the current magnitudes of the two unfed L-probes and the two suspended L-probes are much lower than those on the feed probes. The current distributions on the feed network realized in this manner are shown in Fig. 7.

TABLE II

RADIATION PATTERNS ASSOCIATED WITH EACH DC BIAS

\begin{tabular}{c|c|c|c|c}
\hline \hline DC_broadside & DC_conical & DC_ground\#1 & DC_ground\#2 & Pattern \\
\hline $3 \mathrm{~V}$ & N/A & $0 \mathrm{~V}$ & N/A & Broadside \\
N/A & $3 \mathrm{~V}$ & N/A & $0 \mathrm{~V}$ & Conical \\
\hline \hline
\end{tabular}
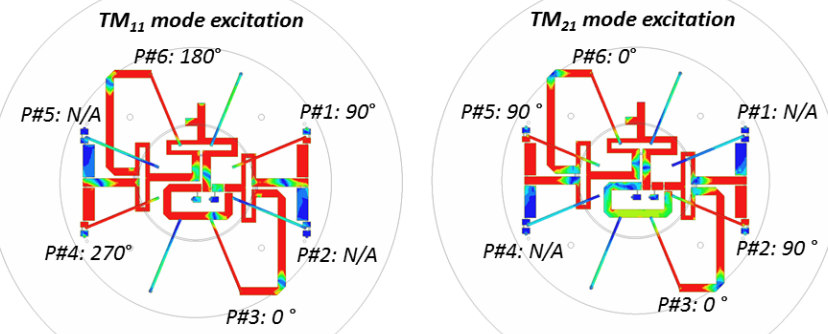

Fig. 7. Current distributions on the reconfigurable feed network when the L-probes are exciting the $\mathrm{TM}_{11}$ and $\mathrm{TM}_{21}$ modes.

\section{Key Design CONSIDERATIONS}

As demonstrated, reconfigurable broadside and conical CP radiation patterns are realized by adopting the reported design strategies. To obtain the optimized radiation performance, two key issues were identified. They are the: (a) width of the annular slot, and (b) effects of the two suspended L-probes.
The width of the annular slot in the circular patch significantly affects the radiation patterns for both modes. Referring to Figs. 4 and 5, we consider two extreme cases: an infinitely thin slot and a very wide slot. If the slot in the patch is infinitely thin, the net result is basically equivalent to the whole patch without any slot. As discussed previously, when the whole patch is present, the $\mathrm{TM}_{21}$ mode is well excited; but the $\mathrm{TM}_{11}$ mode is not at the desired operating frequency. The radiation patterns in broadside mode for different slot widths confirm this conclusion as shown in Fig. 8 (a). If the slot width is very thin, $0.1 \mathrm{~mm}$, the peak of the co-pol LHCP gain is tiny because the $\mathrm{TM}_{11}$ mode is only weakly excited. However, when the width increases from 0.1 to $1.5 \mathrm{~mm}$, the co-pol gain rapidly increases corresponding to the fact that the $\mathrm{TM}_{11}$ mode becomes increasingly more excited. On the other hand, the effect of the slot width on the $\mathrm{TM}_{21}$ mode behavior is different. As seen in Fig. 8 (b), when the slot width increases from 0.1 to $1.5 \mathrm{~mm}$, the resulting conical patterns become more asymmetrical. The $1.5 \mathrm{~mm}$ slot width causes a $2.0 \mathrm{~dB}$ difference in the pattern. This outcome occurs because the large slot will deteriorate the $\mathrm{TM}_{21}$ mode current distribution. Consequently, there is a tradeoff when optimizing the responses from the two modes. To achieve a good balance, the annular slot width was chosen to be $0.5 \mathrm{~mm}$ in the final design.

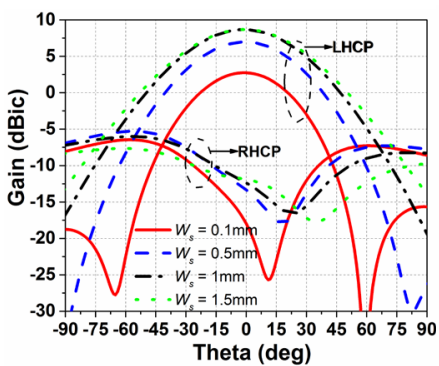

(a)

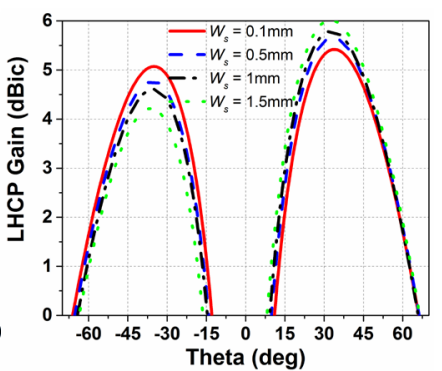

(b)
Fig. 8. Radiation patterns for $\varphi=0^{\circ}$ at $2.5 \mathrm{GHz}$ when the (a) $\mathrm{TM}_{11}$ (broadside) and (b) $\mathrm{TM}_{21}$ modes (conical) are excited.

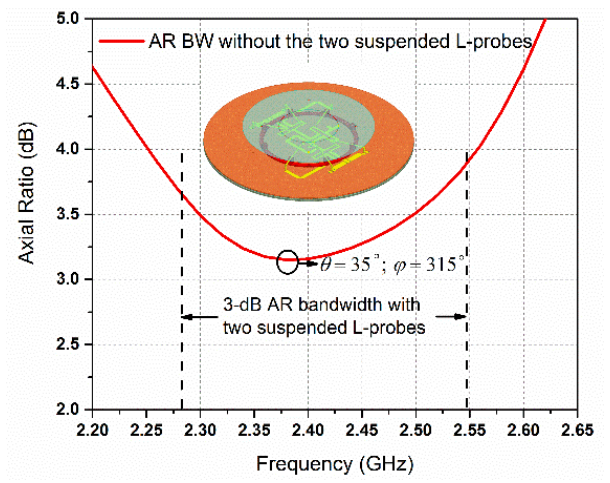

Fig. 9. Comparison of the simulated AR bandwidths with and without the presence of the two suspended L-probes.

The second major design consideration was the use of the two suspended L-probes. Although they are not excited for either the $\mathrm{TM}_{11}$ or the $\mathrm{TM}_{21}$ modes, the two suspended L-probes are arranged to maintain the symmetry of the structure and, hence, to achieve good CP radiation performance. Fig. 9 compares the simulated AR bandwidth with and without the presence of the two suspended Lprobes. Without these passive L-probes, even the best AR values, which occur for $\varphi=135^{\circ}$ at $\theta=35^{\circ}$ across the operational frequency band, are larger than $3 \mathrm{~dB}$. Therefore, the two suspended L-probes are truly indispensable in this design. 


\section{Measured Results}

The components of the optimized design were fabricated and assembled. The resulting prototype reconfigurable $\mathrm{CP}$ antenna is shown in Fig. 10. The L-probes feed and DC biases are indicated in the photos. The four plastic posts that were used to mechanically support the three substrate boards are apparent. The 3D-printed Lprobe-support fixture that anchors the eight L-probes is clearly displayed. The box containing the two $1.5 \mathrm{~V}$ AAA batteries that were used to provide the voltage for DC biasing is also apparent. In the photo, the DC_conical and DC_ground $\# 2$ are biased at $3 \mathrm{~V}$ and $0 \mathrm{~V}$, respectively. As the result, the conical $\mathrm{CP}$ radiation pattern is generated from the displayed configuration. Simply changing the two DC connectors switches the pattern.

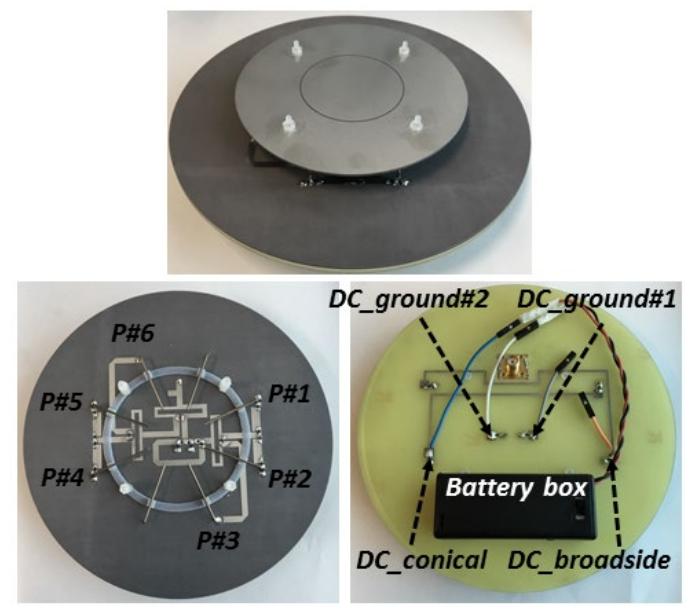

Fig. 10. Photos of the fabricated prototype of the optimized reconfigurable $\mathrm{CP}$ antenna design.

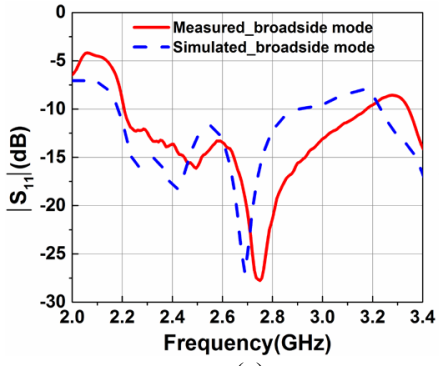

(a)

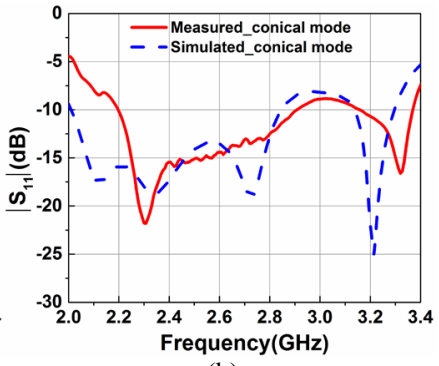

(b)
Fig. 11. Comparison of the measured and simulated reflection coefficients obtained when the (a) $\mathrm{TM}_{11}$ (broadside) and (b) $\mathrm{TM}_{21}$ modes (conical) are excited.

The radiation patterns of the prototype were measured in a SATIMO Starlab near-field measurement system. The reflection coefficients were measured with a Keysight Vector Network Analyzer. A comparison of the measured and simulated reflection coefficients is shown in Fig. 11. The measured overlap of the bandwidths associated with both modes is from 2.2 to $2.9 \mathrm{GHz}$, in good agreement with its simulated value. It should be noted that the measured frequency band moved a bit to higher frequencies than predicted. This is mainly due to the fabrication tolerances, uncertainties in the characteristics of the PCB materials, and discrepancies in the actual device values of the PIN diodes.

Figure 12 presents the measured AR bandwidth for both modes of operation. The overlapping AR bandwidth for both of them is $7.8 \%$ from 2.45 to $2.65 \mathrm{GHz}$. The elevation angle $\theta=35^{\circ}$ is chosen for this determination since this is the angle at which the maximum co-pol gain occurs. The measured AR bandwidth for the conical mode is confined to be within the two curves: $\varphi=270^{\circ}$ and $315^{\circ}$. The AR values within the bandwidth in the other vertical planes are lower than the two presented planes. It is also observed that the measured AR bandwidth has moved to higher frequencies, which is consistent with the reflection coefficient results.

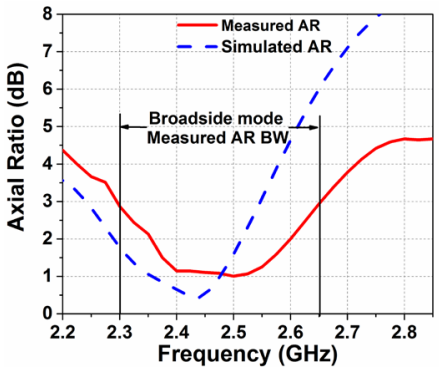

(a)

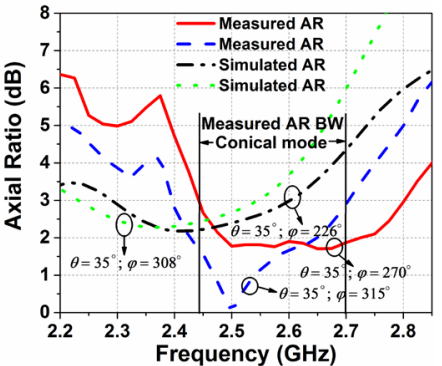

(b)
Fig. 12. Comparison of the measured and simulated axial ratio (AR) bandwidths obtained when the (a) $\mathrm{TM}_{11}$ (broadside) and (b) $\mathrm{TM}_{21}$ modes (conical) are excited.
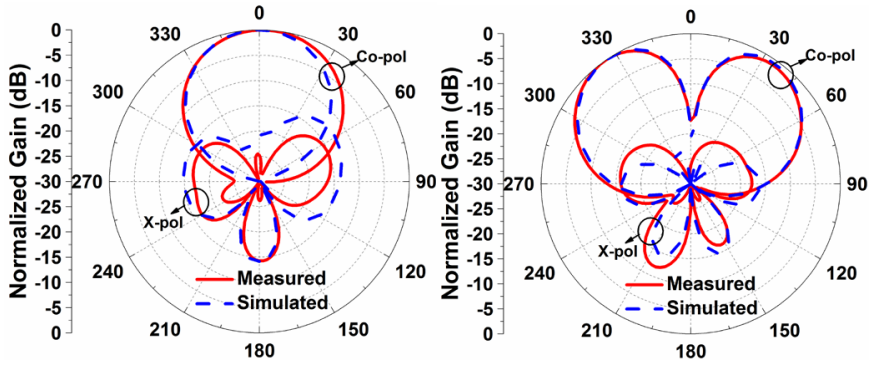

(a) $\varphi=0^{\circ}$ plane
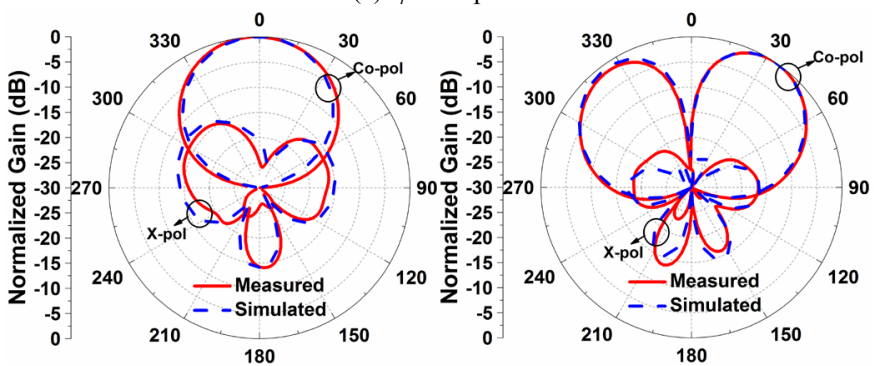

(b) $\varphi=90^{\circ}$ plane

Fig. 13. Measured and simulated realized gain patterns for both the broadside and conical modes at $2.5 \mathrm{GHz}$.

The measured and simulated realized gain patterns for both the broadside and conical modes at $2.5 \mathrm{GHz}$ are shown in Fig. 13. The measured results agree well with their simulated values. Very good switchable broadside and conical $\mathrm{CP}$ gain patterns were realized. The combined AR beamwidth from both modes in the $\varphi=0^{\circ}$ and $90^{\circ}$ planes is $\pm 55^{\circ}$ and $\pm 60^{\circ}$, respectively. The measured results clearly show that the realized gain pattern of the broadside CP mode can successfully cover the null in the corresponding pattern of the conical CP mode. Consequently, they demonstrate that the porotype antenna is a good candidate for the satellite applications presented in Fig. 1. The measured peak gain values are also given in Fig. 14 as functions of the source frequency. Except for a slight frequency shift, the measured peak gains agree well with their simulated values. The maximum gain is $8.5 \mathrm{dBic}$ for the broadside mode and is $5.8 \mathrm{dBic}$ for conical mode. Note that because an omni-directional gain pattern is produced by the conical mode, its peak value is lower than that of the uni-directional gain pattern produced by the broadside mode. 


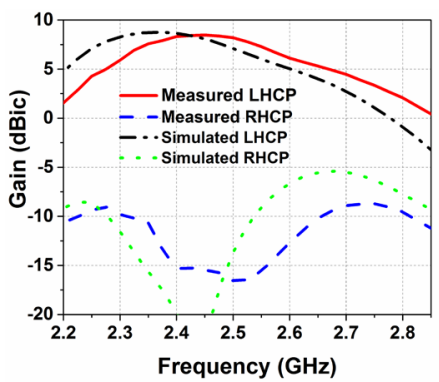

(a)

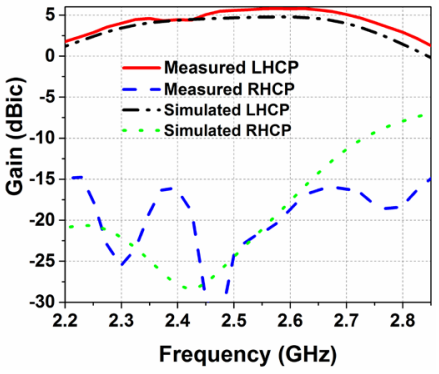

(b)
Fig. 14. Comparison of the measured and simulated peak gain values obtained when the (a) $\mathrm{TM}_{11}$ (broadside) and (b) $\mathrm{TM}_{21}$ modes (conical for $\theta=$ $35^{\circ}, \varphi=90^{\circ}$ ) are excited, as functions of the source frequency.

TABLE III

Comparson of Broadside, Conical Beam Reconfigurable Antennas

\begin{tabular}{c|c|c|c|c|c}
\hline \hline Ref. & Pol. & $\begin{array}{c}\text { BW } \\
(\%)\end{array}$ & $\begin{array}{c}\text { Peak RG } \\
(\mathrm{dBi})\end{array}$ & $\begin{array}{c}\text { Total Eff. } \\
(\%)\end{array}$ & Total Size $\left(\lambda_{0}{ }^{3}\right)$ \\
\hline$[13]$ & LP & 2.5 & $6.8,2.5$ & 75,60 & $0.67 \times 0.67 \times 0.05$ \\
\hline$[14]$ & LP & 6.6 & $6.5,4.0$ & 87,45 & $0.88 \times 0.88 \times 0.06$ \\
\hline$[15]$ & LP & 6.5 & $5.3,2.6$ & 68,76 & $0.65 \times 0.69 \times 0.06$ \\
\hline$[16]$ & LP & 9.0 & $4.1,4.8$ & NA & $0.51 \times 0.39 \times 0.01$ \\
\hline$[17]$ & CP & 1.6 & $4.0,2.0$ & 68,79 & $1.05 \times 1.05 \times 0.04$ \\
\hline Ours & CP & 7.8 & $8.5,5.8$ & 78,82 & $1.15 \times 1.15 \times 0.12$ \\
\hline
\end{tabular}

Table III summarizes the measured characteristics of the broadside and conical beam pattern-reconfigurable antennas reported to date in the literature. It was determined that only two systems: the one in [17] and the one we reported here, are designed to achieve the desired reconfigurable $\mathrm{CP}$ patterns. Referring to these tabulated results, our design achieves superior performance characteristics when compared to the system reported in [17]. The realized operating bandwidth, $7.8 \%$, is 4.8 times wider; and the peak realized gain $(\mathrm{RG})$ values for the broadside and conical modes $(8.5 \mathrm{dBic}$ and $5.8 \mathrm{dBic}$ ) are $4.5 \mathrm{~dB}$ and $3.8 \mathrm{~dB}$ higher, respectively. These enhancements were achieved with an increase in the height (from $0.04 \lambda_{0}$ to $0.12 \lambda_{0}$ ) of the realized system, but only a slight increase in the lateral size (from $1.05 \lambda_{0}$ to $1.15 \lambda_{0}$ ).

\section{CONCLUSION}

A pattern-reconfigurable annular slot-augmented circular patch antenna with switchable broadside and conical CP beams was reported. Two key design strategies, the slot-augmented circular patch and the mode switchable L-probe based feed network, were described in detail. These critical design concepts and their optimizations were discussed. It was demonstrated that the slot augmentation of the circular patch led to the overlap of the operational bandwidths of both the $\mathrm{TM}_{11}$ and $\mathrm{TM}_{21}$ modes and that the feed network facilitated their proper excitation. The $\mathrm{TM}_{11}\left(\mathrm{TM}_{21}\right)$ mode was shown to produce the broadside (conical) beam. The fabrication and testing of a prototype of the optimized design were also reported. The measured and simulated performance characteristics were shown to be in reasonable agreement, thus validating these design strategies. It was further demonstrated that this realized system outperforms the only other design reported in its class and has measured performance characteristics which make it a good candidate for satellite communications and other wireless systems where broad CP coverage is highly desired.

\section{ACKNOWLEDGEMENT}

The authors would like to thank Prof. Y. Jay Guo from the University of Technology Sydney (UTS) for his valuable comments pertaining to the work reported in this paper. In addition, they want to thank Mr. Shulin Chen from UTS and Mr. Xin Huang from Xiamen University for their help with the measurements of the prototype. The authors would also like to thank Mr. C. K Lau from the City University of Hong Kong for the fabrication of the L-shaped probes.

\section{REFERENCES}

[1] Y. Zhou, R. S. Adve, and S. V. Hum, "Design and evaluation of pattern reconfigurable antennas for MIMO applications," IEEE Trans. Antennas Propag., vol. 62, no. 3, pp. 1084-1092, Mar., 2014.

[2] C. Kittiyanpunya and M. Krairiksh, "A four-beam pattern reconfigurable Yagi-Uda antenna," IEEE Trans. Antennas Propag., vol. 61, no. 12, pp. 6210-6214, Dec., 2013.

[3] W. Kang, S. Lee, and K. Kim, "Design of symmetric beam pattern reconfigurable antenna," Electron. Lett., vol. 46, no. 23, pp. 1536-1537, Nov., 2010.

[4] J. Ren, X. Yang, J. Y. Yin, and Y. Z. Yin, "A novel antenna with reconfigurable patterns using H-shaped structures," IEEE Antenna Wirel. Propag. Lett., vol. 14, pp. 915-918, 2015.

[5] W. Lin and H. Wong, "Pattern reconfigurable wideband circularlypolarized quadrifilar helix with broadside and backfire radiation patterns," 9th European Conference on Antennas and Propagation (EuCAP), Lisbon, 2015.

[6] S. V. Hum, M. Okoniewski, and R. J. Davies, "Modeling and design of electronically tunable reflectarrays," IEEE Trans. Antennas Propag., vol. 55, no.8, pp. 2200-2210, Aug., 2007.

[7] M. Riel and J. J. Laurin, "Design of an electronically beam scanning reflectarray using aperture-coupled elements," IEEE Trans. Antennas Propag., vol. 55, no.5, pp. 1260-1266, May, 2007.

[8] A. Tayebi, J. Tang, P. R. Paladhi, L. Udpa, S. S. Udpa, and E. J. Rothwell, "Dynamic beam shaping using a dual-band electronically tunable reflectarray antenna," IEEE Trans. Antennas Propag., vol. 63, no.10, pp. 4534-4539, Oct., 2015.

[9] D. Sievenpiper, J. Schaffner, R. Loo, and G. Tangonan, "Reconfigurable antennas based on electrically tunable impedance surfaces," in Proc. 31st European Microwave Conference, London, UK, Sep. 2001.

[10] J. D. Boerman and J. T. Bernhard, "Performance study of pattern reconfigurable antennas in MIMO communication systems," IEEE Trans. Antennas Propag., vol. 56, no. 1, pp. 231-236, Jan. 2007.

[11] H. Kawakami, G. Sato, and R. Wakabayashi, "Research on circularly polarized conical-beam antennas," IEEE Antennas Propag. Mag. , vol. 39, no. 3, pp. 27-39, Jun. 1997

[12] W. Lin and H. Wong, "Circularly polarized conical beam antenna with very thin profile and wide bandwidth," IEEE Trans. Antennas Propag., vol. 62, no. 12, pp. 5974-5982, Dec. 2014.

[13] S. H. Chen, J. S. Row, and K. L. Wong, "Reconfigurable square-ring patch antenna with pattern diversity," IEEE Trans. Antennas Propag., vol. 55, no.2, pp. 472-475, Feb. 2007.

[14] P. Y. Qin, Y. J. Guo, A. R. Weily, and C. H. Liang, "A pattern reconfigurable U-slot antenna and its applications in MIMO systems," IEEE Trans. Antennas Propag., vol. 60, no.2, pp. 516-528, Feb. 2012.

[15] W. S. Kang, J. A. Park, and Y. J. Yoon, "Simple reconfigurable antenna with radiation pattern," Electron. Lett., vol. 44, no.3, Jan. 2008.

[16] I. Lim and S. Lim, "Monopole-like and boresight pattern reconfigurable antenna," IEEE Trans. Antennas Propag., vol. 61, no.12, pp. 58545859, Dec. 2013.

[17] N. R. Labadie, S. K. Sharma, and G. M. Rebeiz, "A circularly polarized multiple radiating mode microstrip antenna for satellite receive applications," IEEE Trans. Antennas Propag., vol. 62, no.7, pp. 34903500 , July 2014.

[18] J. Huang, "Circularly polarized conical patterns from circular microstrip antenna," IEEE Trans. Antennas Propag., vol. AP-32, no.9, pp. 991994, Sep. 1984.

[19] W. Lin and H. Wong, "Polarization reconfigurable aperture-fed patch antenna and array," IEEE Access, vol. 4, pp. 1510-1517, Apr. 2016.

[20] Data Sheet of Bar50-02L PIN Diodes, Infineon Technologies, Application Note [Online]. Available: http:// www.infineon.com/.

[21] Data Sheet of LQW18AN56NJ00 inductor, Murata Manufacturing Co., Ltd., Application Note [Online]. Available: http:// www.murata.com/. 